\title{
Bioprocess intensification: an aqueous two-phase process for the purification of C-phycocyanin from dry Spirulina platensis
}

\author{
Li Zhao $\cdot$ Yi-liang Peng · Jia-mei Gao $\cdot$ Wei-min Cai
}

Received: 5 August 2013 / Revised: 29 October 2013 / Accepted: 30 October 2013 / Published online: 17 November 2013

(C) The Author(s) 2013. This article is published with open access at Springerlink.com

\begin{abstract}
The dry Spirulina powders are rich in nutritional compounds especially including C-phycocyanin (C-PC) have been principal raw material in the food processing. But the purity of C-PC in the dry powders was not up to the food grade standard. In this study, C-PC was recovered and purified from the dry algae powders using aqueous twophase system (ATPS). The optimal conditions were proved in polyethylene glycol (PEG) 1000 and sodium phosphate, system $\mathrm{pH}$ of 5.8 , the tie-line length of $28.50 \%(\mathrm{w} / \mathrm{w})$ and the volume ratio $(\mathrm{Vr})$ of 0.16 to increase the purity from the initial purity of 0.42 to 1.31 after the first extraction. The recovery yield was $89.52 \%$. After the third ATPS extraction, the purity and the purification factor were achieving up 2.11 and 5.01. It was successfully decreased the viscosity of the system and extraction time by application of PEG 1000. It facilitated the feasibility of the scaling-up in industry.
\end{abstract}

Keywords Aqueous two-phase systems ·

C-phycocyanin $\cdot$ Dry powders $\cdot$ Purity $\cdot$ Recovery

\section{Introduction}

Phase separation in solutions containing polymer mixtures is a very common phenomenon. In the aqueous two-phase

\author{
L. Zhao $\cdot$ W. Cai $(\square)$ \\ School of Municipal and Environmental Engineering, Harbin \\ Institute of Technology, Harbin 150090, China \\ e-mail:wwwbbll@sina.com \\ L. Zhao \\ e-mail: lili_zhao_cc@126.com \\ L. Zhao $\cdot$ Y. Peng $\cdot$ J. Gao \\ College of Life Sciences and Biotechnology, Harbin Normal \\ University, Harbin 150025, China
}

system (ATPS), the bulk of both phases consists of water, and ATPS forms a gentle environment for biomaterials [1]. This technique has been characterized for low cost and easy to scale up. Furthermore, the polymers were known to have a stabilizing influence on the particle structures and the biological activities [2]. So ATPS has advantages over conventional extraction using organic solvents. It has been a very useful separation tool for a variety of applications [3], especially of biomaterials, including plant and animal cells, microorganisms, proteins and nucleic acids [4-6]. In ATPS, the partitioning of the desired proteins to one phase and contaminant proteins to the other phase not only purifies the proteins but also concentrates them in one water phase.

The C-phycocyanin (C-PC) is a kind of blue-colored protein, which has great commercial and industrial significance. It is formed by two subunits of $\alpha$ and $\beta$ with the molecular weight of 18.0 and $20.0 \mathrm{kDa}$, respectively. It has been not only widely used in foods and cosmetics [7], but also used as fluorescent marker in the biomedical research and as therapeutic agent in oxidative stress-induced diseases [8]. The purity of 0.7 is considered as food grade, 3.9 as reactive grade and over 4.0 as analytical grade [9]. The commercial values of the food grade and the analytical grade have been reported approximately as high as US\$0.13 and US\$50 per mg. The Spirulina platensis contains over $20 \% \mathrm{C}$-PC, so it was always used as raw material to extract the C-PC [10].

Because of the industrial and commercial value of the C-PC, various researchers have developed several methods of the purification previously. But these methods have been characterized by high cost, lots of stages and low recovery [11]. Then the scaling-up of these processes was difficult and expensive. Using of ATPS to separate the C-PC has been an attractive alternative to overcome the disadvantages. 
Previous many researchers have applied ATPS to the separation of phycobiliprotein [12-14]. But most of them applied the ATPS with ion exchange chromatography or others in the purification processes [12]. There was only one study which applied the aqueous two-phase extraction for the purification of the C-PC with the optimized conditions of PEG 4000 and potassium phosphate system [15] in the past two decades. However, the majority of C-PC was gathered in the PEG phase. The viscosity of the PEG was increased with the increasing of the molecular weight of the PEG. There was a hindrance to the selective removal of the PEG. So to use the low molecular weight was important for the purification processes. The PEG-potassium phosphate system at the $\mathrm{pH} 6.0$ showed best results in the terms of purity. But the stock solution of potassium phosphate was easy to crystallize at this $\mathrm{pH}$ value. The sodium phosphate solution was stable at the $\mathrm{pH} 5.8$, which was higher than the isoelectric point (pI) value of the C-PC is 4.8 [16]. All the studies on obtaining the C-PC were to separate them from the fresh algae. But the fresh algae had traces of active algae toxins. The dry algae powders were safer, which were processed to meet requirement of the food grade before they were purchased from the foodstuff factory. Furthermore, neither time nor energy was spent on cultivating algae in the processes. The dry algae powders were favorable to scaling-up and applying in industry.

In view of this, the main objective of the present study is to establish an efficient, simple and commercial downstream process to recover the primary of the C-PC from the dry algae powder of S. platensis.

\section{Materials and methods}

The dry powders of Spirulina platensis

The dry powders of S. platensis were procured from Ocean University of China. The cell morphology of the algae powders was observed with the light microscope.

The crude extraction of the C-PC

The dry algae powders of $S$. platensis were frozen at $-20{ }^{\circ} \mathrm{C}$ and were dissolved at $4{ }^{\circ} \mathrm{C}$ for four times with the distilled water. The biomass was centrifuged at $8,000 \mathrm{rpm}$ for about $10 \mathrm{~min}$ to separate the C-PC from the cell and stored at $4{ }^{\circ} \mathrm{C}$ for further use.

Aqueous two-phase systems

\section{The composition of the aqueous two-phase systems}

The protein purification in polymer-salt phase was conducted. The binodal curves were estimated by the turbidimetric titration method using the PEG of the molecular weight (MW) of $600,1,000,1,500,2,000,4,000$ and $6,000(50 \%(\mathrm{w} / \mathrm{w})$ stock solution) and dipotassium hydrogen phosphate/potassium dihydrogenphosphate, sodium dihydrogen phosphate/disodium hydrogen phosphate, sodium sulfate and ammonium sulfate $(30 \%(\mathrm{w} / \mathrm{w})$ stock solution). Fine adjustment of $\mathrm{pH}$ was made by addition of orthophosphoric acid or sodium hydroxide. Predetermined quantities of the stock solutions of the PEG and the salts were mixed with the crude extract of the C-PC to make the total weight of the system $100 \%$ on w/w basis. The mixture was in vibration thoroughly for about $10 \mathrm{~min}$ to equilibrate.

\section{The parameter of ATPS for the purification of the C-PC}

The tie-line length (TLL) represents the length of the line connects the composition of the top and bottom phase of the ATPS. It is often used to express the effect of system composition on partitioned material, where the $\mathrm{TLL}=\left(\Delta C_{\mathrm{T}}^{2}+\Delta C_{\mathrm{B}}^{2}\right)^{1 / 2}, \Delta C_{\mathrm{T}}$ denotes the difference in concentration of component top phase polymer between top and bottom, and $\Delta C_{\mathrm{B}}$ denotes similarly the difference in concentration of component bottom phase salt.

The volumes of the phases were used to estimate the volume ratio $(\mathrm{Vr})$. The visual estimates of the volumes of top and bottom phases were made in graduate tubes.

The partition coefficient $(\mathrm{K})$ was the ratio of the concentration of solute in the top phase to that in the bottom phase. The C-PC exhibited a strong preference to the top phase. The concentration of the C-PC in the top was $0.16 \mathrm{mg} / \mathrm{ml}$ and in the bottom was $0.000284 \mathrm{mg} / \mathrm{ml}$ (data not shown). So the bottom phase recovery was not estimated.

\section{Multiple Aqueous two-phase extraction}

The subsequent ATPS stages were composed of the top PEG phase from the previous extraction and the fresh bottom phase of the same composition as the first extraction. The operating conditions of the subsequent process were kept constant and were similar to those defined for the first extraction.

\section{Analytical procedures}

The absorption spectra of the C-PC were recorded by using a UV-vis spectrophotometer at the room temperature. The experiments were performed in triplicate. The purity of the C-PC was defined as the relation between of 620- and 280$\mathrm{nm}$ absorbance. The purification factor was defined as the increase in the purity of the C-PC which is relative to that of the initial purity of crude extract. The yield was defined as the top phase protein recover. Results reported are the average of three independent experiments and standard 
deviation. All the figures were prepared by EXCEL2000, ORIGN7.0 and MATLAB R2008a.

\section{Ultrafiltration}

The top phase was recovered by ultrafiltration with the membrane of $30 \mathrm{kDa}$ at $10,000 \mathrm{rpm}$ for $10 \mathrm{~min}$ to remove the PEG.

The bioactivity of the C-PC

The bioactivity of the C-PC was recorded by the fluorescence emission spectrum. The excitation wavelength of the C-PC fluorescence was from 400 to $700 \mathrm{~nm}$.

The qualitative analysis of the C-PC

The qualitative analysis of the C-PC was performed by the sodium dodecyl sulfate polyacrylamide gel electrophoresis (SDS-PAGE) [15].

\section{Results and discussion}

The morphology of the algae powders

The cellular morphology of the algae powder was observed with the light microscope. Most cells were spiral with one half of whorls (Fig. 1). The initial purity of the C-PC was 0.42 .

The influence of phase forming salt and PEG molecular weight

In order to select suitable salt for the purification of the C-PC, the ATPS was carried out at the Vr 1.0 and TLL

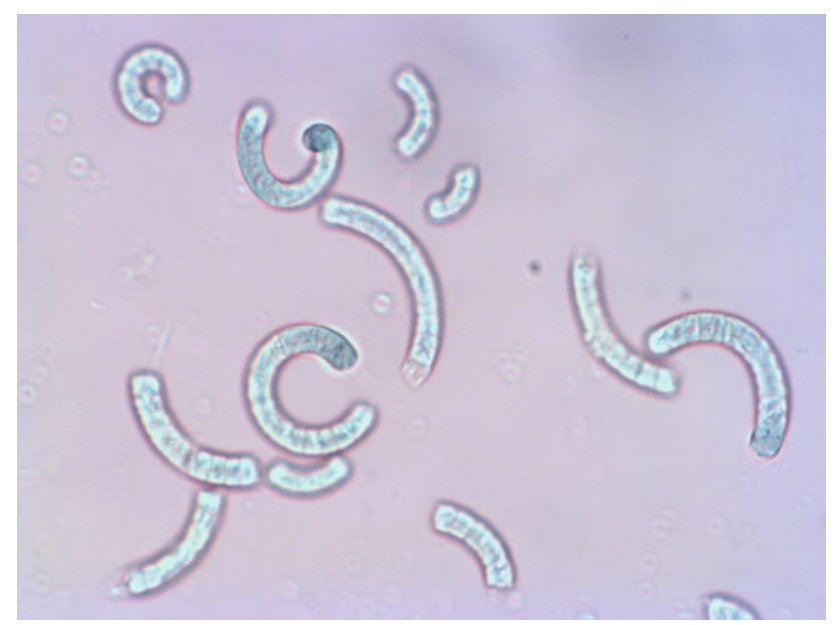

Fig. 1 The morphology of the dry Spirulina platensis powders

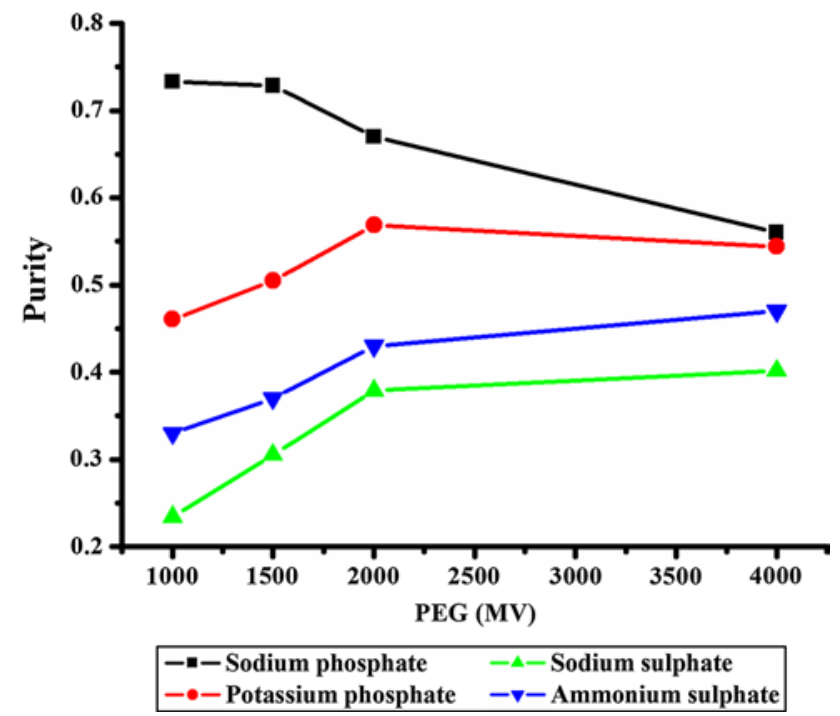

Fig. 2 The influence of different salts on the purity of the C-PC

$28 \pm 1 \%$ by adding different salts (ammonium sulfate, sodium sulfate, sodium phosphate and potassium phosphate), different molecular weight PEG (Mw 1,000, 1,500, 2,000 and 4,000) and a given quantity of the crude extract of the C-PC which makes the total weight of the system $100 \%$ on w/w basis. The results were shown in Fig. 2. It was clear that the purity of the PEG-sodium phosphate system was higher than the other systems. The isoelectric point (pI) value of the C-PC is 4.8. Ammonium sulfate, sodium sulfate, sodium phosphate and potassium phosphate gave the $\mathrm{pH}$ of 4.8, 5.0, 5.8 and 6.8, respectively. The C-PC was hydrophilic [17]. The initial system $\mathrm{pH}$ value generated electrochemical affinity between negatively charged products and the PEG. It was necessary to select the initial $\mathrm{pH}$ value that was more than $\mathrm{pI}$ value. The phosphates were suitable for the purification. And PEG-phosphate system was often chosen for the purification because it was referred a biocompatible phase environment for the C-PC. But the stock solution of potassium phosphate was easy to crystallize at low $\mathrm{pH}$ value. The sodium phosphate solution was stable at the $\mathrm{pH} 5.8$, which was higher than the $\mathrm{pI}$ value of the C-PC. Furthermore, the PEG-sodium phosphate system showed the best results of purity with the different PEG molecular weight from 1,000 to 4,000. Therefore, the sodium phosphate was chosen for further experiments.

The influence of PEG molecular weight

In order to select suitable molecular weight of the PEG, ATPS was performed with different molecular weights of PEG, which were $600,1,000,1,500,2,000,4,000$ and 6,000 . The phase composition was of the PEG $13 \%$ and sodium phosphate $14 \%$ at room temperature. The results 


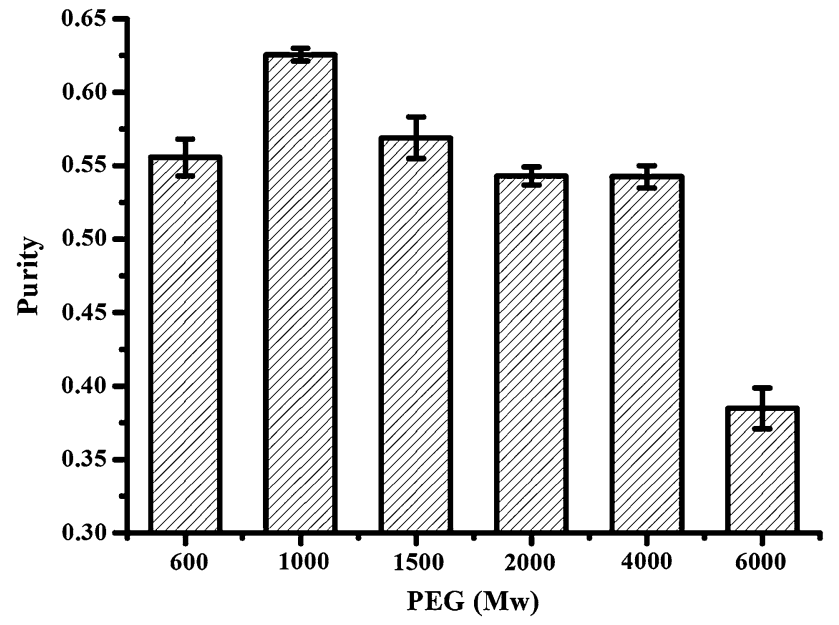

Fig. 3 The influence of different molecular weights of PEG on the purity of the C-PC

were shown in Fig. 3. It was clear that the partition purity of PEG 1000 was higher than the other molecular weights of PEG systems. The PEG has a positive dipolar momentum with terminal hydroxyl groups. At the same concentration of the polymer, the molecular weight of PEG was decreased with more hydroxyl groups, so the polar increased, which caused the increase in the hydrophilic. The C-PC is a highly hydrophilic protein with the molecular weight of 44,000 $\mathrm{g} / \mathrm{mol}$ [18]. Hence, the decreasing PEG molecular weight was favored the hydrophilic C-PC to the top PEG phase. On the other hand, the molecular weight of PEG was decreased with less viscosity, which caused the increase in the free volume, meaning less resistance and more space available for the protein. Furthermore $\mathrm{Vr}$ increasing revealed more yield with the declining PEG molecular weight at the same PEG composition in the system. In view of this, the PEG 1000 was chosen for further experiments.

The influence of TLL and Vr

It has been observed that the hydrophilic protein of high molecular weight $(>10,000 \mathrm{~g} / \mathrm{mol})$ was favored when both low Mw PEG $(<4,000 \mathrm{~g} / \mathrm{mol})$ and low or medium TLL $(<40 \% \mathrm{w} / \mathrm{w})$ [19]. So the ATPS was performed with different TLL (\% w/w) of 20.74, 25.05, 28.50, 30.46 and 33.11 at different $\mathrm{Vr}$ from 0.16 to 2.67. The results were shown in Fig. 4a, b. The temperature and the $\mathrm{pH}$ of the system were kept constant. The TLL was the critical factor of the ATPS, which was used to express the effect of system composition on partitioned material. And the time of phase separation depended on the distance of the working tie-line from the critical. The maximum purity of 1.31 was observed at $\mathrm{Vr}$ of 0.16 in TLL of $28.50 \%$. The influence
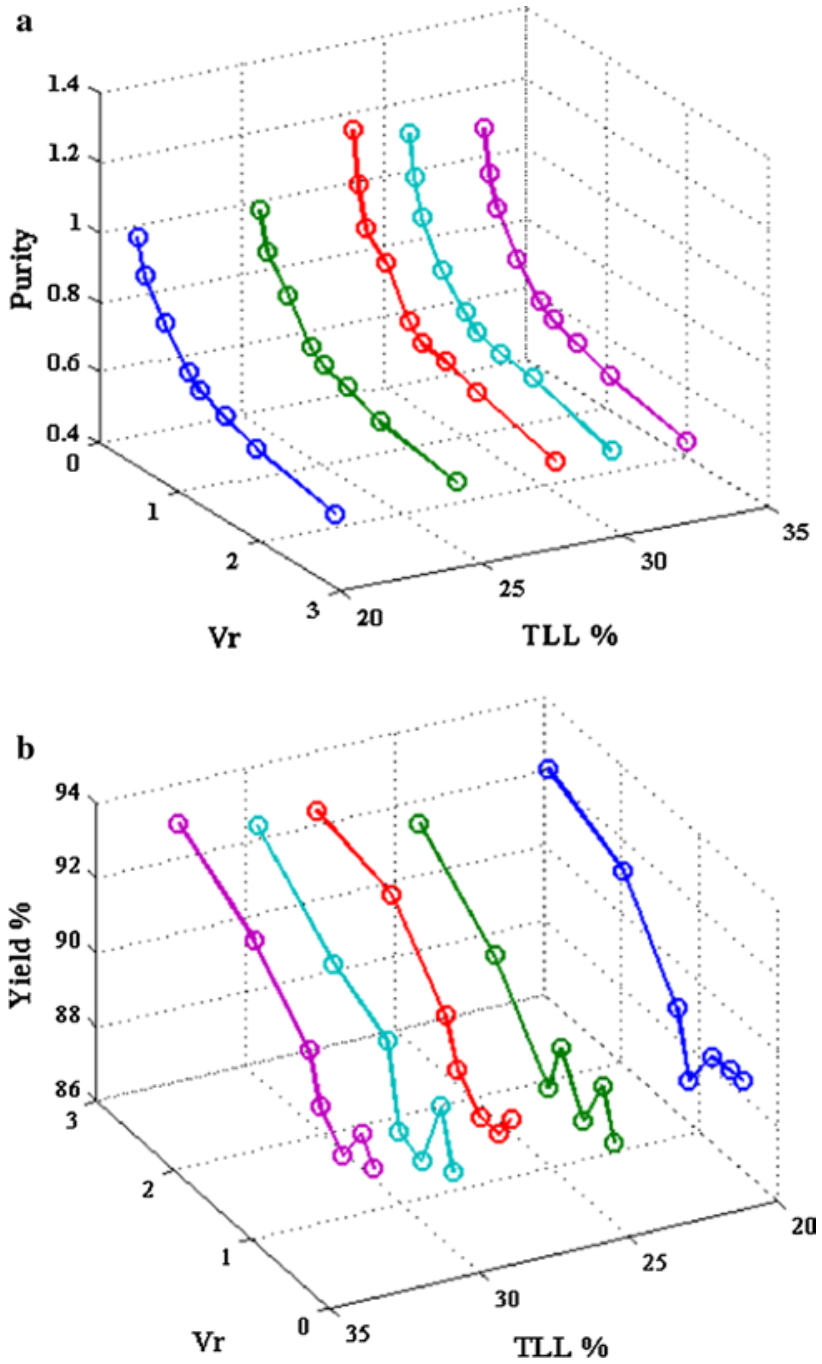

Fig. 4 a The influence of different $\mathrm{Vr}$ and TLL on the purity of the C-PC. $\mathbf{b}$ The influence of different $\mathrm{Vr}$ on the yield of the C-PC

of TLL on the purity and yield of the PC was not striking, but the influence of $\mathrm{Vr}$ was striking. It was clear that the purity of the C-PC was increased with the decrease in $\mathrm{Vr}$ at the each TLL. It was possible that the less space available for the total protein in the top phase. So the contaminant proteins had partitioned to the bottom phase with increment in the volume in the bottom phase. The algae exhibited the same partition behavior [20]. But it exhibited an opposite behavior of the fresh algae with the PEG of 4000 [15]. It might be explained that the molecular weight of the PEG affected the hydrophobic of the ATPS. The concentration was increasing with decreasing Vr. But the volume of top phase was decreasing with decreasing Vr. It was clear that the maximum yield of the C-PC was at the $\mathrm{Vr}$ of 2.67 in the range of 92-94\% for all TLL. The yield was decreasing with the decrease in $\mathrm{Vr}$ from 1 to 2.67, and it fluctuated according to the $\mathrm{Vr}$ in the range from 87 to $90 \%$ when the 


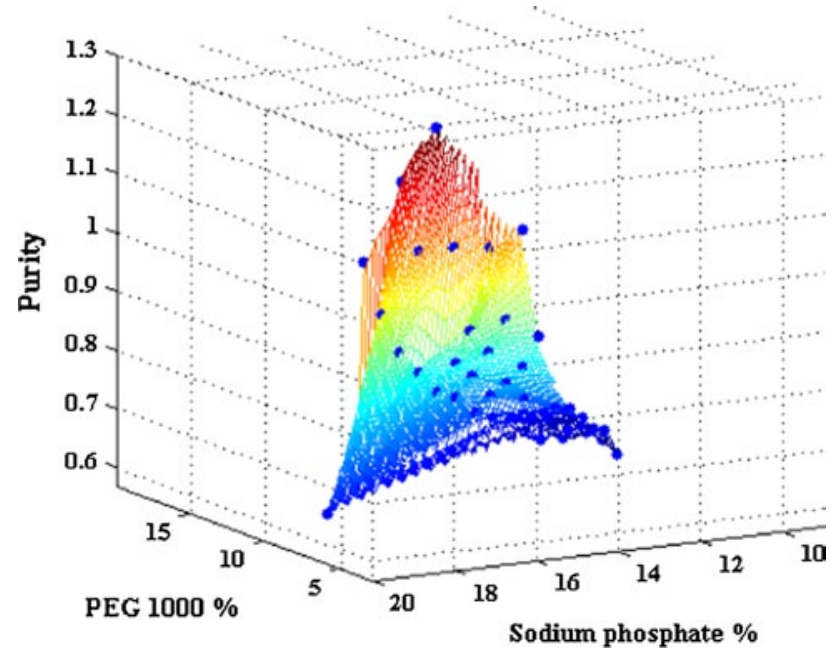

Fig. 5 The influence of components in the phase on the purity of the C-PC

$\operatorname{Vr}$ was $<1$ for all TLL. The influence of TLL and Vr on the yield of P-PC was not striking.

The composition concentration in a single step of ATPS

In order to select the optimum composition concentration, the ATPS was performed with different formulations of PEG (Mw 1,000) and sodium phosphate at different TLL. Other parameters such as the temperature and the $\mathrm{pH}$ of the system were kept constant. The results were shown in Fig. 5. The optimum components were PEG $10004.22 \%$ $(\mathrm{w} / \mathrm{w})$ and sodium phosphate $18 \%(\mathrm{w} / \mathrm{w})$ with TLL $28.50 \%, \mathrm{Vr} 0.16$ and $\mathrm{pH} 5.8$, which the maximum purity of the C-PC was 1.31 , the yield was $89.52 \%$ and the purification factor was 3.12. It was completely different from the fresh algae, which the optimum components were PEG 4000 and potassium phosphate system with parameters of TLL $35.53 \%$, Vr 0.8, pH 6, the yield $85.68 \%$ and the purification factor was 2.98 [15]. The optimum composition concentrations of PEG 1000 and sodium phosphate were lower than those of the fresh, because the binodal of the sodium phosphate system was lower than that of the potassium phosphate system. It was meaning that using less chemical medicine to achieve the blue-colored protein. It facilitated the feasibility of the scaling-up in industry.

The influence of multiple aqueous two-phase extraction

In order to increase the purity of the C-PC, the multiple aqueous two-phase extraction was carried out. The purification factors for the C-PC were shown in Fig. 6. It was clear that the purification factor of the third extraction was the highest. The first ATPS was carried out in PEG 1000

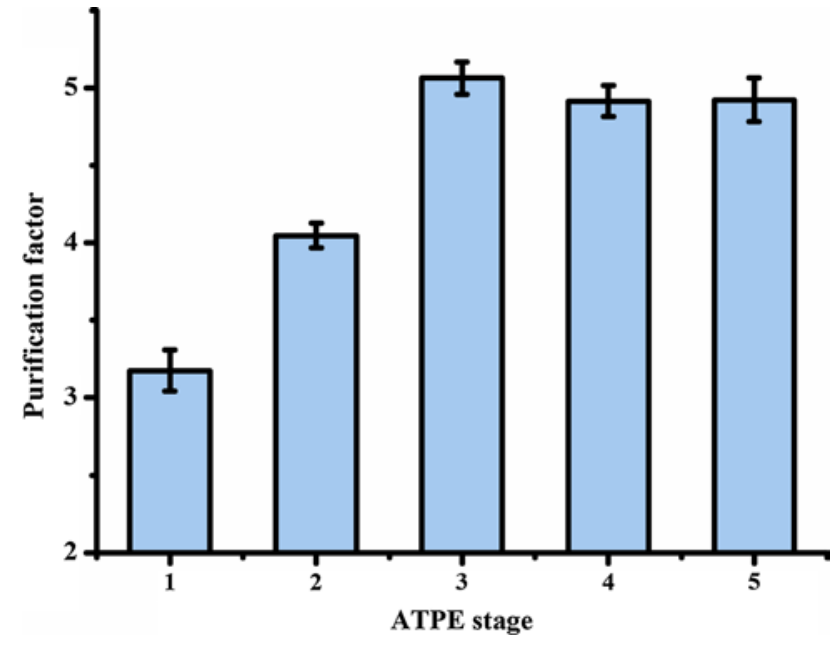

Fig. 6 The influence of multiple ATPE stages on the purification factor of the C-PC

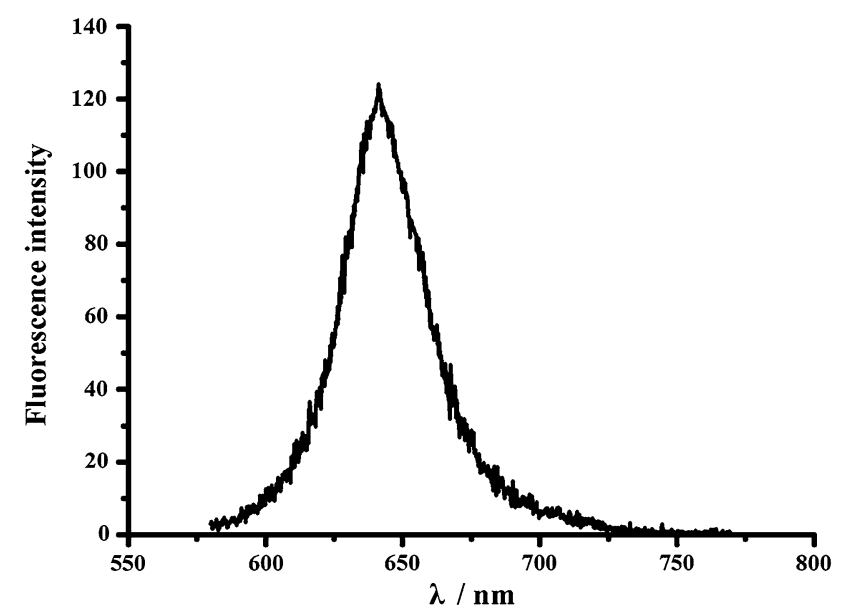

Fig. 7 The fluorescence emission spectrum of the recovery C-PC

and sodium phosphate system with the TLL $28.50 \%$, the Vr 0.16 and the system pH 5.8. It seemed that the consecutive ATPS caused the purity of the top phase to increase from 0.42 to 2.11 after the third extraction. The purification factor was 5.01. Further ATPS stage did not increase in the purity of the C-PC.

\section{Ultrafiltration}

The purity of the C-PC remained constant after ultrafiltration, but the concentration of the C-PC increased.

The bioactivity of the C-PC

The fluorescence emission spectrum of the recovery C-PC was shown in Fig. 7. Fluorescence emission spectra were 


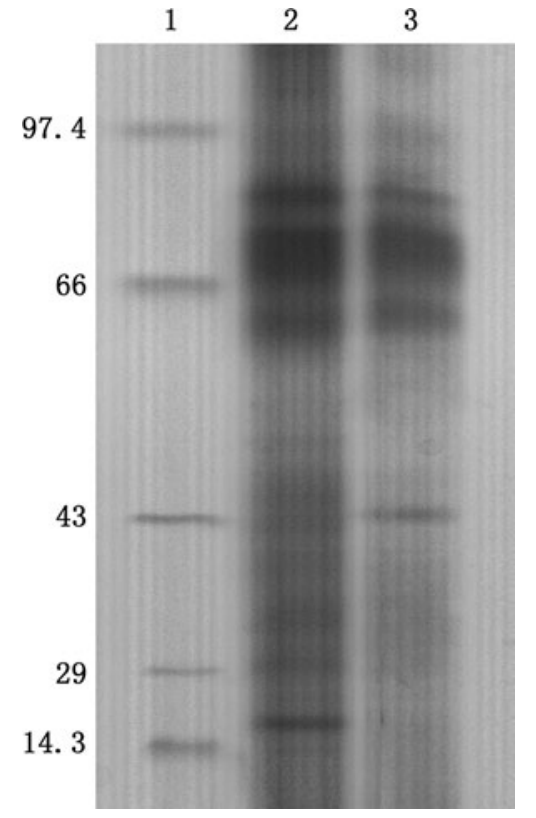

Fig. 8 The SDS-PAGE of the C-PC

excited at $560 \mathrm{~nm}$ and recorded from 580 to $770 \mathrm{~nm}$ (excitation slit was $0.2 \mathrm{~nm}$ ). The wave crests of the C-PC fluorescence emission spectrum were in 641 and $642 \mathrm{~nm}$. It proved that the bioactivity of the recovery of the C-PC was well.

The qualitative analysis of the C-PC

The qualitative analysis of the C-PC was performed by the SDS-PAGE as show in Fig. 8.

The lane 1 indicated the molecular maker, the lane 2 indicated the crude extract of the C-PC and the lane 3 indicated the C-PC after the third ATPS extraction. It was observed that the band of $44 \mathrm{kDa}$ was visible in the lane 3 . It was clearer than that in the lane 2 . Hence, the purity of the C-PC increased after ATP extraction. There were some contaminant proteins of high molecular weight which were different from the fresh algae [15].

\section{Conclusions}

This study reports a systematic approach of ATPS for the recovery and purification of the C-PC from the dry S. platensis powder. The optimal conditions were proved in the PEG 1000 and sodium phosphate, the system $\mathrm{pH}$ of 5.8 , the TLL of $28.50 \%$ and the $\mathrm{Vr}$ of 0.16 to increase the purity from 0.42 to 1.31 . The product yield was $89.52 \%$. The third ATP extraction resulted in further increase in the purity of 2.11 , and the purification factor was 5.01 , if increasing the initial purity of the crude extraction would achieve more purity of the C-PC by the systematic bioprocess of ATPS.

Acknowledgments The authors would like to acknowledge the testing center of Harbin Normal University.

Conflict of interest None.

Compliance with Ethics Requirements This article does not contain any studies with human or animal subjects.

Open Access This article is distributed under the terms of the Creative Commons Attribution License which permits any use, distribution, and reproduction in any medium, provided the original author(s) and the source are credited.

\section{References}

1. Marrcos JC, Fonseca LP, Ramalho MT, Cabral JMS (2002) Application of surface response analysis to the optimization of penicillin acylase purification in aqueous two-phase systems. Enzyme Microb Technol 31:1006-1014

2. Kepka C, Collet E, Persson J, Stahl A (2003) Pilot-scale extraction of an intracellular recombinant cutinase from E. coli cell homogenate using a thermoseparating aqueous two-phase system. J Biotechnol 103:165-181

3. Naganagouda K, Mulimani VH (2008) Aqueous two-phase extraction (ATPE): an attractive and economically viable technology for downstream processing of Aspergillus oryzae $\alpha$-galactosidase. Process Biochem 43:1293-1299

4. Jain A, Johri BN (1999) Partitioning of an extracellular xylanase produced by a thermophilic fungus Melanocarpus albomyces IIS-68 in an aqueous two-phase system. Bioresour Technol 67:205-207

5. Show PL, Tan CP, Shamsul AM, Ariff A, Yusof YA, Chen SK, Ling TC (2012) Extractive fermentation for improved production and recovery of lipase derived from Burkholderia cepacia using a thermoseparating polymer in aqueous two-phase systems. Bioresour Technol 116:226-233

6. Garza-Madrid M, Rito-Palomares M, Serna-Saldívar SO, Benavides J (2012) Potential of aqueous two-phase systems constructed on flexible devices: human serum albumin as proof of concept. Process Biochem 45:1082-1087

7. Yoshida A, Takagaki Y, Nishimune T (1996) Enzyme immunoassay for phycocyanin as the main component of spirulina color in foods. Biosci Biotechnol Biochem 60:57-60

8. Bhat VB, Madyastha KM (2001) Scavenging of peroxynitrite by phycocyanin and phycocyanobilin from Spirulina platensis: protection against oxidative damage to DNA. Biochem Biophys Res Commun 285:262-266

9. Rito-Palomares M, Nunez L, Amador D (2001) Practical application of aqueous two-phase systems for the development of a prototype process for c-phycocyanin recovery from Spirulina maxima. J Chem Technol Biotechnol 76:1273-1280

10. Ogbonda KH, Aminigo RE, Abu GO (2007) Influence of temperature and $\mathrm{pH}$ on biomass production and protein biosynthesis in a putative Spirulina sp. Bioresour Technol 98:2207-2211

11. Ranjitha K, Kaushik BD (2005) Purification of phycobiliproteins from Nostoc muscorum. Sci Ind Res 64:372-375

12. Patil G, Chethana S, Sridevi AS, Raghavarao KSMS (2006) Method to obtain C-phycocyanin of high purity. Chromatogr A 1127:76-81

13. Kula MR, Kroner KH, Hustedt H (1982) Purification of enzymes by liquid-liquid extraction. Adv Biochem Eng 24:73-118 
14. Diamond AD, Hsu JT (1992) Aqueous two-phase systems for biomolecule separation. Adv Biochem Eng/Biotechnol 47:89-135

15. Patil G, Raghavarao KSMS (2007) Aqueous two phase extraction for purification of C-phycocyanin. Biochem Eng J 34:156-164

16. MacColl R, Lee JJ, Berns DS (1971) Protein aggregation in C-Phycocyanin. Studies at very low concentrations with the photoelectric scanner of the ultracentrifuge. Biochem J 122:421-426

17. Benavides J, Rito-Palomares M (2008) Review practical experiences from the development of aqueous two-phase processes for the recovery of high value biological products. J Chem Technol Biotechnol 83:133-142

18. Ciferri O, Tiboni O (1985) The biochemistry and industrial potential of Spirulina. Ann Rev Microbiol 39:503-526

19. Cabezas H (1996) Theory of phase formation in aqueous two phase systems. Chromatogr B 680:3-30

20. Benavides J, Palomares MR (2005) Potential aqueous two-phase processes for the primary recovery of colored protein from microbial origin. Eng Life Sci 3:259-266 